\title{
Front Matter: Volume 9605
}

, "Front Matter: Volume 9605," Proc. SPIE 9605, Techniques and Instrumentation for Detection of Exoplanets VII, 960501 (26 October 2015); doi: $10.1117 / 12.2218637$

EDIE Event: SPIE Optical Engineering + Applications, 2015, San Diego, California, SPIE. United States 


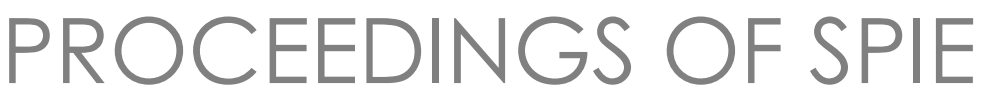

\section{Techniques and Instrumentation for Detection of Exoplanets VII}

\section{Stuart Shaklan}

Editor

10-13 August 2015

San Diego, California, United States

Sponsored and Published by

SPIE 
The papers in this volume were part of the technical conference cited on the cover and title page. Papers were selected and subject to review by the editors and conference program committee. Some conference presentations may not be available for publication. Additional papers and presentation recordings may be available online in the SPIE Digital Library at SPIEDigitallibrary.org.

The papers reflect the work and thoughts of the authors and are published herein as submitted. The publisher is not responsible for the validity of the information or for any outcomes resulting from reliance thereon.

Please use the following format to cite material from these proceedings:

Author(s), "Title of Paper," in Techniques and Instrumentation for Detection of Exoplanets VII, edited by Stuart Shaklan, Proceedings of SPIE Vol. 9605 (SPIE, Bellingham, WA, 2015) Six-digit Article CID Number.

ISSN: 0277-786X

ISSN: 1996-756X (electronic)

ISBN: 9781628417715

Published by

SPIE

P.O. Box 10, Bellingham, Washington 98227-0010 USA

Telephone +1 3606763290 (Pacific Time) · Fax +1 3606471445

SPIE.org

Copyright (C) 2015, Society of Photo-Optical Instrumentation Engineers.

Copying of material in this book for internal or personal use, or for the internal or personal use of specific clients, beyond the fair use provisions granted by the U.S. Copyright Law is authorized by SPIE subject to payment of copying fees. The Transactional Reporting Service base fee for this volume is $\$ 18.00$ per article (or portion thereof), which should be paid directly to the Copyright Clearance Center (CCC), 222 Rosewood Drive, Danvers, MA 01923. Payment may also be made electronically through CCC Online at copyright.com. Other copying for republication, resale, advertising or promotion, or any form of systematic or multiple reproduction of any material in this book is prohibited except with permission in writing from the publisher. The CCC fee code is 0277-786X/15/\$18.00.

Printed in the United States of America.

Publication of record for individual papers is online in the SPIE Digital Library.

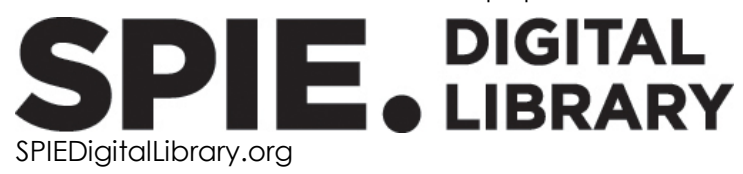

Paper Numbering: Proceedings of SPIE follow an e-First publication model. A unique citation identifier (CID) number is assigned to each article at the time of publication. Utilization of CIDs allows articles to be fully citable as soon as they are published online, and connects the same identifier to all online and print versions of the publication. SPIE uses a six-digit CID article numbering system structured as follows:

- The first four digits correspond to the SPIE volume number.

- The last two digits indicate publication order within the volume using a Base 36 numbering

system employing both numerals and letters. These two-number sets start with 00, 01, 02, 03, 04, 05, 06, 07, 08, 09, 0A, OB ... 0Z, followed by 10-1Z, 20-2Z, etc. The CID Number appears on each page of the manuscript. 


\title{
Contents
}

\author{
vii Authors \\ xi Conference Committee
}

WFIRST/AFTA I

960502 Requirements and design reference mission for the WFIRST/AFTA coronagraph instrument [9605-1]

960504 The WFIRST/AFTA coronagraph instrument optical design [9605-3]

960505 An overview of WFIRST/AFTA coronagraph modeling [9605-4]

960506 Effect of DM actuator errors on the WFIRST/AFTA coronagraph contrast performance [9605-5]

960507 Wavefront correction with Kalman filtering for the WFIRST-AFTA coronagraph [9605-6]

WFIRST/AFTA II

960509 Low order wavefront sensing and control for WFIRST-AFTA coronagraph [9605-8]

9605 OA Shaped pupil Lyot coronagraph designs for WFIRST/AFTA [9605-9]

9605 OB Laboratory performance of the shaped pupil coronagraphic architecture for the WFIRST/AFTA coronagraph [9605-10]

WFIRST/AFTA III

9605 OE The impact of radiation damage on photon counting with an EMCCD for the WFIRST-AFTA coronagraph [9605-13]

9605 OG Prototype imaging spectrograph for coronagraphic exoplanet studies (PISCES) for WFIRST/AFTA [9605-15]

HIGH CONTRAST LABORATORY RESULTS

$9605 \mathrm{OH}$ Studies of the effects of control bandwidth and dark-hole size on the $\mathrm{HClT}$ contrast performance [9605-16]

9605 Ol High-contrast imager for complex aperture telescopes (HiCAT): 3. first lab results with wavefront control [9605-17] 
9605 OJ EXCEDE technology development IV: demonstration of polychromatic contrast in vacuum at $1.2 \mathrm{~N} / \mathrm{D}$ [9605-18]

9605 OK Demonstrating broadband billion-to-one contrast with the Visible Nulling Coronagraph [9605-19]

9605 OL Exoplanet coronagraph shaped pupil masks and laboratory scale star shade masks: design, fabrication and characterization [9605-20]

\section{WAVEFRONT CONTROL AND SIGNAL EXTRACTION}

9605 OM Active correction of aperture discontinuities (ACAD) for space telescope pupils: a parametic analysis [9605-21]

9605 ON Hybrid Lyot coronagraph for WFIRST-AFTA: coronagraph design and performance metrics [9605-22]

960500 Estimation of chromatic errors from broadband images for high contrast imaging [9605-23]

9605 OP Preliminary analysis of effect of random segment errors on coronagraph performance [9605-24]

9605 OR Blind source separation approaches for exoplanet signal extraction [9605-26]

9605 OS Data processing and algorithm development for the WFIRST-AFTA coronagraph: reduction of noise free simulated images, analysis and spectrum extraction with reference star differential imaging [9605-27]

EXO-C PROBE STUDY

9605 OT Exo-C: a probe-scale space observatory for direct imaging and spectroscopy of extrasolar planetary systems (Invited Paper) [9605-28]

$96050 \mathrm{~V} \quad$ PIAA coronagraph design for the Exo-C Mission concept [9605-30]

EXO-S PROBE STUDY

9605 OW The Exo-S probe class starshade mission (Invited Paper) [9605-31]

$96050 \mathrm{X}$ Optical instrumentation for science and formation flying with a starshade observatory [9605-32]

9605 OY Design reference missions for the exoplanet starshade (Exo-S) probe-class study [9605-33]

$96050 Z$ Error budgets for the Exoplanet Starshade (Exo-S) probe-class mission study [9605-34]

960511 Design of a laboratory testbed for external occulters at flight Fresnel numbers [9605-84] 
960512 Fully achromatic nulling interferometer (FANI) for high SNR exoplanet characterization [9605-37]

960514 A pareto-optimal characterization of miniaturized distributed occulter/telescope systems [9605-39]

960515 Maturing CCD photon-counting technology for space flight [9605-75]

SPACE-BASED IMAGING AND TRANSIT

960517 How to directly image a habitable planet around Alpha Centauri with a $\sim 30-45 \mathrm{~cm}$ space telescope [9605-41]

960518 Orbital Differential Imaging: a new high-contrast post-processing technique for direct imaging of exoplanets [9605-42]

960519 The low-order wavefront sensor for the PICTURE-C mission [9605-43]

9605 IA End-to-end simulation of high-contrast imaging systems: methods and results for the PICTURE mission family [9605-44]

9605 1B The CHEOPS instrument on-ground calibration system [9605-45]

\section{GROUND-BASED INSTRUMENTS AND PROCESSING}

9605 IC The CHARIS IFS for high contrast imaging at Subaru [9605-46]

9605 1D First light with ALES: A 2-5 micron adaptive optics Integral Field Spectrograph for the LBT [9605-47]

\section{GROUND-BASED INSTRUMENTS AND PROCESSING II}

9605 1G Exoplanet science with the LBTI: instrument status and plans [9605-50]

$960511 \quad$ Optimized focal and pupil plane masks for vortex coronagraphs on telescopes with obstructed apertures [9605-52]

9605 1J A Mach-Zehnder interferometer based on orbital angular momentum for improved vortex coronagraph efficiency [9605-53]

9605 1L ExTrA: Exoplanets in transit and their atmospheres [9605-55] 
9605 IP Archival Legacy Investigations of Circumstellar Environments (ALICE): Statistical assessment of point source detections [9605-59]

POSTER SESSION

9605 1Q Fluoride fiber thermal emission study for SPIRou @ CFHT [9605-60]

9605 1R A new fiber slit assembly for the FOCES spectrograph [9605-61]

9605 IT A white super-stable source for the metrology of astronomical photometers [9605-63]

$96051 \mathrm{D}$ Design of the ilocater acquisition camera demonstration system [9605-64]

9605 IV Numerically designed phase-mask for stellar coronagraph [9605-65]

9605 IW Sparse aperture mask for low order wavefront sensing [9605-66]

9605 IY High-contrast coronagraph performance in the presence of DM actuator defects [9605-68]

960522 Design of off-axis PIAACMC mirrors [9605-72]

960524 PISCES: high contrast integral field spectrograph simulations and data reduction pipeline [9605-74]

960525 Technological progress of a ferrofluid deformable mirror with tunable nominal optical power for high-contrast imaging [9605-76]

960528 Zernike wavefront sensor modeling development for LOWFS on WFIRST-AFTA [9605-79]

960529 Deconvolution of differential OTF (dOTF) to measure high-resolution wavefront structure [9605-80]

9605 2A UA wavefront control lab: design overview and implementation of new wavefront sensing techniques [9605-81]

9605 2B Adaptive optics self-calibration using differential OTF (dOTF) [9605-82]

$96052 \mathrm{C}$ Control design for momentum-compensated fast steering mirror for WFIRST-AFTA coronagraph instrument [9605-83]

$96052 \mathrm{E} \quad$ Scaling relation for occulter manufacturing errors [9605-86]

96052 F Astrometric accuracy of aperture making interferometry with JWST-NIRISS [9605-87]

$96052 \mathrm{G}$ A method to directly image exoplanets in multi-star systems such as Alpha-Centauri [9605-88]

$960521 \quad$ Initial look at the coronagraph technology gaps for direct imaging of exo-earths [9605-90] 


\section{Authors}

Numbers in the index correspond to the last two digits of the six-digit citation identifier (CID) article numbering system used in Proceedings of SPIE. The first four digits reflect the volume number. Base 36 numbering is employed for the last two digits and indicates the order of articles within the volume. Numbers start with 00, 01, 02, 03, 04, 05, 06, 07, 08, 09, OA, OB...0Z, followed by 10-1Z, 20-2Z, etc.

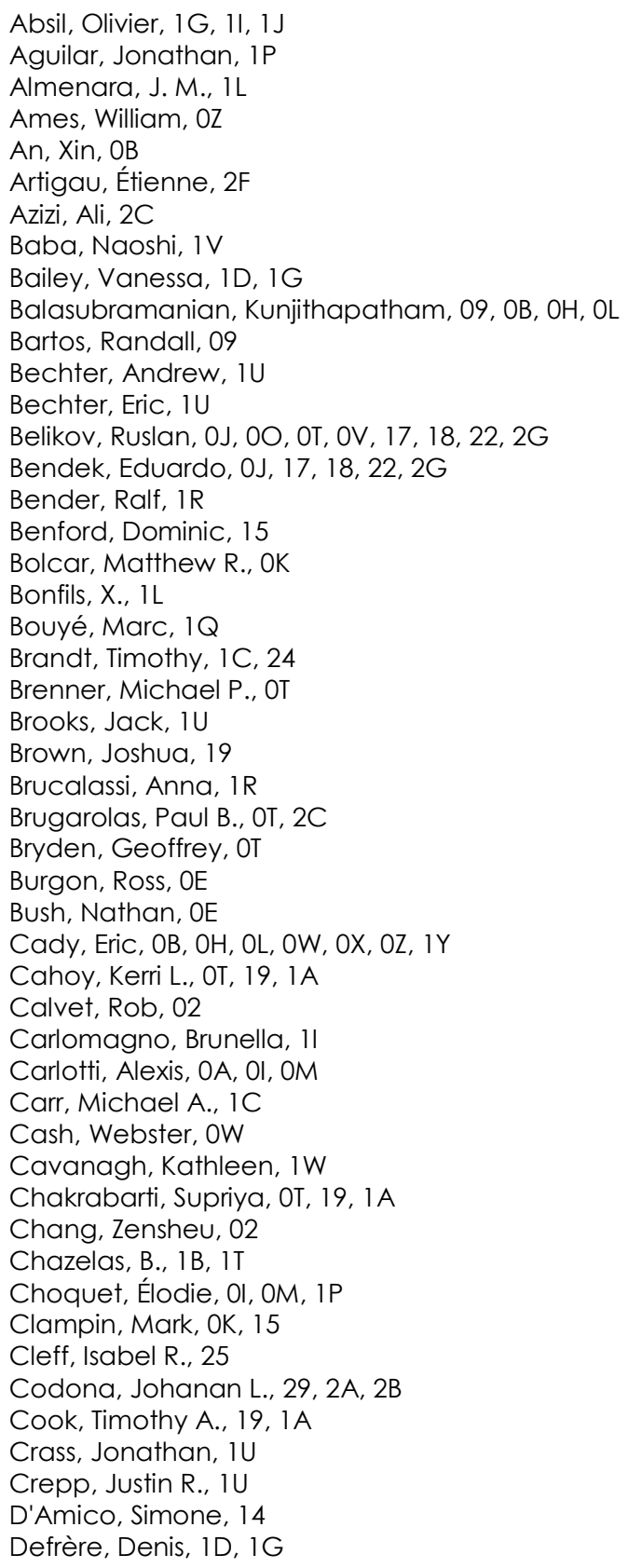

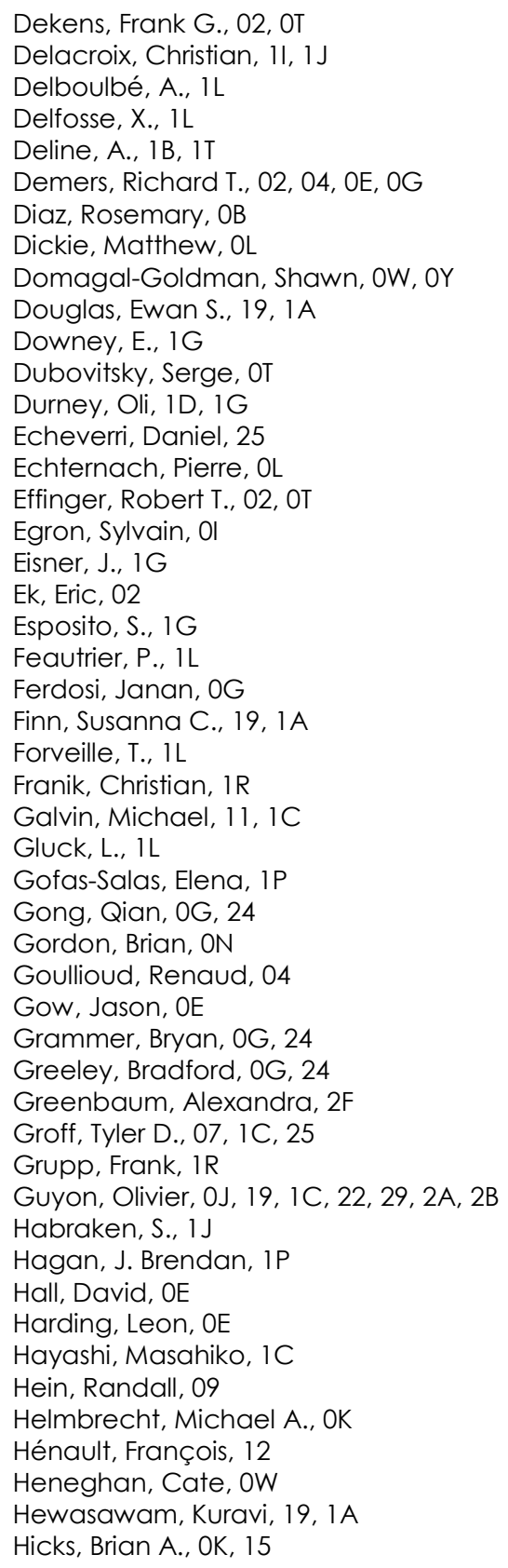


Hill, J. M., $1 G$

Hilton, George, 0G, 24

Hinz, Philip, 1D, 1G, $1 \mathrm{U}$

Hirsch, Brian, OT

Hix, Troy, OJ

Hoenk, Michael, OE

Hoffmann, W. F., 1G

Holland, Andrew, OE

Hopp, Ulrich, IR

Hovland, Larry, 02

Howe, Glenn A., 19, 1 A

Huby, Elsa, 1I, $1 \mathrm{~J}$

Jarosik, Norman, $1 \mathrm{C}$

Jocou, L., $1 \mathrm{~L}$

Jones, Laura, 02

Jordan, Douglas, OE

Jovanovic, Nemanja, $1 \mathrm{C}$

Karlsson, M., $1 \mathrm{~J}$

Kasdin, N. Jeremy, 07, OA, OB, OL, OW, 11, 1C, 1W,

25, 2E

Kellermann, Hanna, 1R

Kenworthy, M., $1 G$

Kern, Brian, 09, OB, OG, OL

Kern, P., $1 \mathrm{~L}$

Ketterer, Ryan, $1 \mathrm{U}$

Kim, Ki-Won, 11

Kim, Sug-Whan, 11

Kim, Yunjong, 11

King, David, $1 \mathrm{U}$

Kissil, Andrew, OT

Knapp, Gillian, 1C

Knight, Justin M., 29, 2A, 2B

Koenig, Adam W., 14

Kouach, Driss, $1 Q$

Krist, John E., 04, 05, 09, ON, OT, OV

Kuchner, Marc J., OW, IA

Kuhnert, Andreas, OB

Lafrasse, S., $1 \mathrm{~L}$

Lajoie, Rachel, 0 I

Lam, Raymond, 09

Lang, Jared J., OT

Lang-Bardl, Florian, IR

Leboulleux, Lucie, 이

Leisenring, Jarron, 1D, 1G

Lemmer, Aaron J., 25

Levecq, Olivier, 01

Leviton, Douglas B., 1C

Lewis, Nikole K., $1 \mathrm{~A}$

Liebe, Carl, OX

Limbach, Mary Anne, 1C

Lisman, P. Douglas, OW, OY, OZ

Llop Sayson, Jorge, 0G, 24

Loc, Anthony, 02

Long, Chris A., Ol

Loomis, Craig, $1 \mathrm{C}$

Lozi, Julien, 0J, 17, 19

Lynch, Dana H., OJ

Lyon, Richard G., OK, 15

Macintosh, Bruce, OS, 14
Magnard, Y., $1 \mathrm{~L}$

Males, Jared R., 17, 18

Mallik, Udayan, OK, 15

Mandic, Milan, 2C

Marchen, Luis, $\mathrm{OZ}$

Marinan, Anne D., 19, 1A

Marley, Mark S., OT

Martel, André, 2F

Martel, Jason, 19

Martin, Stefan R., OW, OX, OZ

Marx, Catherine, 0G, 24

Maurel, D., 1L

Mawet, Dimitri, 0M, 19, 1A, 11, 1J

Mazoyer, Johan, OI, OM

McElwain, Michael W., OG, OT, 15, 1C, 24

McMahon, T., $1 \mathrm{G}$

Meadows, Victoria S., OT

Mede, Kyle, 1C

Mejia Prada, Camilo, OB, OL

Memarsadeghi, Nargess, OG, 24

Mendillo, Christopher B., 19, 1A

Mennesson, B., $1 G$

Michaels, Darren, $\mathrm{OE}$

Micheau, Yoan, 1Q

Millan-Gabet, R., $1 G$

Miller, Ian J., OK

Miller, Kelsey L., 29, 2A, 2B

Miller, Kevin H., 1C

Miura, Noriaki, $1 \mathrm{~V}$

Montoya, Manny, 1D, 1G

Moody, Dwight, ON

Moore, Douglas, 09

Moore, James, 09

Morgan, Rhonda, 2l

Moulin, T., 1L

Muller, Richard, OL

Murakami, Naoshi, $1 \mathrm{~V}$

Murgas, F., 1L

Murray, Neil, OE

N'Diaye, Mamadou, Ol, OM

Nelson, M., $1 G$

Nelson, Matt, 1D

Nemati, Bijan, 02, 05, OB, OE

Neville, Timothy, 02

Nissen, Joel, OT

Noecker, Charley, 02

Norman, Colin, OM

Oseas, Jeffrey M., OT

Parisot, Jérôme, $1 Q$

Patterson, Keith, 09, OB, 2C

Peddada, Pavani, OE

Perrin, Marshall D., OG, OI, OM, OS, 1P, 24

Petrone, Peter III, OK, 15

Pham, Hung, 02

Piron, Pierre, $11,1 \mathrm{~J}$

Pluzhnik, Eugene, 0J, 22

Poberezhskiy, llya, 09, 0B, OL

Pong, Chris, OT

Pueyo, Laurent, Ol, OM, OS, IP 
Puglisi, A., $1 G$

Quijada, Manuel A., 1C

Rabou, P., $1 \mathrm{~L}$

Rajan, Abhijith, IP

Regehr, Martin, $\mathrm{OZ}$

Reynolds, Robert, $1 \mathrm{U}$

Riggs, A. J. Eldorado, 07, OA, OB, OL, IW

Roberge, Aki, OW, OY

Rochat, S., 1L

Rodack, Alexander T., 29, 2A, 2B

Roux, A., $1 \mathrm{~L}$

Ruane, Garreth J., 11, 1 J

Rud, Mayer, 02, 04

Ryan, Daniel, OB, OL

Ryu, Dongok, 11

Sarajlic, M., 1B

Savransky, Dmitry, OR

Scharf, Daniel, OW, OX

Schneider, Glenn, 0J

Seager, Sara, OW

Serabyn, Eugene, OT

Shaklan, Stuart B., OH, OL, OP, OW, OY, OZ, 1Y, 2E

Shi, Fang, 04, 06, 09, 28, 2C

Shields, Joel, 09, 2C

Sidick, Erkin, 05, 06, 09, 0H, $1 Y$

Siegler, Nick, 21

Singh, Garima, 19

Sirbu, Dan, 0J, OL, 0O, 11, 2E

Sivaramakrishnan, Anand, $2 F$

Skemer, Andrew J., 1D, 1G

Skrutskie, Michael F., 1D, 1G

Soman, Matthew, OE

Sordet, M., 1B

Soummer, Rémi, Ol, OM, OS, IP

Spalding, E., $1 \mathrm{G}$

Sparks, William, OW

Stadler, E., $1 \mathrm{~L}$

Stahl, H. Philip, OP

Stahl, Mark T., OP

Stapelfeldt, Karl R., OG, OT, OV

Stark, Christopher, OY

Stone, Jordan, 1D, 1G

Subedi, Hari, IW

Sunada, Eric, OT

Surdej, J., $1 \mathrm{~J}$

Swartzlander, Grover A. Jr., 11

Takato, Naruhisa, 1C

Tamura, Motohide, IV

Tang, Hong, 02, 04, 09, 0G, 0X, 2C

Thatte, Deepashri, 2F

Thomas, Sandrine J., 0J, 17, 2G

Thomson, Mark, OW, $\mathrm{OZ}$

Titus, Charles J., 14

Trabert, Rachel, OW, OY

Trauger, John T., ON, OT

Truong, Tuan, 09

Turnbull, Margaret, OW, OY

Unwin, Stephen C., OT

van der Marel, Roeland, OS
Vanderbei, Robert J., OA, 11, 2E

Vaz, A., 1G

Villalvazo, Juan, 02

Wallace, J. Kent, 09, 28

Wang, Xu, 09, 28, 2C

Warfield, Keith R., OT, OW

Webb, David, OW

White, Victor, OL

Wildi, F. P., 1B, $1 T$

Wilson, Dan, 09

Wilson, John, 1D, 1G

Woodward, Charles E., ID

Wunsche, A., $1 \mathrm{~L}$

Yee, Karl, OL

Ygouf, Marie, OS

Zell, Peter, 0J

Zhao, Bo, $1 \mathrm{U}$

Zhao, Feng, 04

Zhou, Hanying, 05, OB, OL

Zimmer, Robert, $\mathrm{OB}$

Zimmerman, Neil T., OA, OB, OL, IW 
Proc. of SPIE Vol. $9605960501-10$

Downloaded From: https://www.spiedigitallibrary.org/conference-proceedings-of-spie on 26 Apr 2023 Terms of Use: https://www.spiedigitallibrary.org/terms-of-use 


\section{Conference Committee}

Program Track Chair

Oswald H. W. Siegmund, University of California, Berkeley

(United States)

Conference Chair

Stuart Shaklan, Jet Propulsion Laboratory (United States)

Conference Program Committee

Olivier Guyon, Subaru Telescope, National Astronomical Observatory of Japan (United States) and Research Corporation of University of Hawaii (United States) and The University of Arizona (United States)

Lucas Labadie, University of Cologne (Germany)

Bruce A. Macintosh, Lawrence Livermore National Laboratory (United States)

Dimitri P. Mawet, California Institute of Technology (United States)

M. Charley Noecker, Jet Propulsion Laboratory (United States)

Rémi Soummer, Space Telescope Science Institute (United States)

\section{Session Chairs}

1 WFIRST/AFTA I

M. Charley Noecker, Jet Propulsion Laboratory (United States)

2 WFIRST/AFTA ॥

M. Charley Noecker, Jet Propulsion Laboratory (United States)

3 WFIRST/AFTA III

Tyler D. Groff, Princeton University (United States)

4 High Contrast Laboratory Results

Stuart Shaklan, Jet Propulsion Laboratory (United States)

5 Wavefront Control and Signal Extraction

Dimitri Mawet, California Institute of Technology (United States)

6 Exo-C Probe Study

Olivier Guyon, Subaru Telescope, National Astronomical Observatory of Japan (United States) and Research Corporation of University of Hawaii (United States) and The University of Arizona (United States) 
7 Exo-S Probe Study

Bruce A. Macintosh, Stanford University (United States)

8 Mission Concepts and Technologies

Dimitri Mawet, California Institute of Technology (United States)

9 Space-Based Imaging and Transit

Tyler D. Groff, Princeton University (United States)

10 Ground-Based Instruments and Processing

Bruce A. Macintosh, Lawrence Livermore National Laboratory (United States)

11 Ground-Based Instruments and Processing II

Bruce A. Macintosh, Stanford University (United States)

12 Ground-Based Instruments and Processing III

Stuart Shaklan, Jet Propulsion Laboratory (United States)

13 Archival NICMOS Data

Stuart Shaklan, Jet Propulsion Laboratory (United States) 